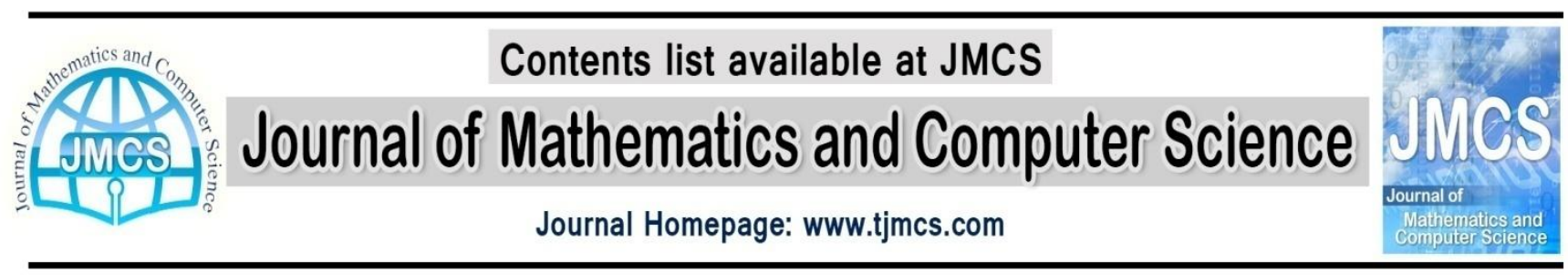

\title{
On Bivariate Haar Functions and Interpolation Polynomial
}

\author{
R. Dehghan, K. Rahsepar Fard \\ Department of Mathematics, Islamic Azad University, Masjed Soleiman branch, Masjed Soleiman, Iran.
}

Department of Computer Engineering, University of Qom, Qom, Iran

Article history:

E-mail: rezadehghanamoli@gmail.com

Received February 2014

Accepted March 2014

Available online March 2014

\begin{abstract}
In this paper we consider bivariate Haar series in general case, where bivariate Haar functions are defined on the plane. Here we define a new bivariate Haar function that is included two independent variables. Indeed we presented the new function that is not in previous researches. Mathematicians have applied bivariate Haar function based on tensor product that is a special case of bivariate case. In this research we define the Haar functions by applying another way. Therefore, we define the Haar function differently. And also, the interpolation polynomial with two variables is explained. Then we compare two methods for calculating the approximating function. Namely, we consider a numerical example for comparing the new approximation to bivariate interpolation polynomial. In this example we compute interpolation polynomial by points with Newton lattice form. The calculations indicate that the accuracy of the obtained solutions is acceptable when the number of calculation points is small.
\end{abstract}

Keywords: Bivariate Haar function, Bivariate interpolation polynomial, Haar Fourier coefficient, Haar series

\section{Introduction}

The wavelets technique allows the creation of very fast algorithms when compared to algorithms which are ordinarily used. Various wavelet basis are applied, we can see some of wavelet applications in [1113]. One of the wavelet basis is Haar wavelet where we use of this kind of base on Bivariate interpolation polynomial.Before defining the Haar system, we introduce the standard notation for binary intervals, which will be used throughout the rest of the paper. A binary interval is an interval of the form

$$
\left(\frac{i-1}{2^{k}}, \frac{i}{2^{k}}\right) \text {, where } i=1,2, \ldots, 2^{k}, k=0,1, \ldots
$$




\section{2. . MATERIALS AND METHODS}

\subsection{Main properties of the Haar System}

The Haar functions are the most elementary wavelets. They still illustrate in the most direct way some of the main features of wavelet decompositions. Here, we shall consider in some detail the properties that make them suitable for numerical applications.

Let $\Omega_{1}$ be the set of $(n, k)$ such that $1 \leq k \leq 2^{n}, n=0,1, \ldots, \quad \Omega:=\Omega_{1} \cup(0,0)$. Denote

$$
\begin{aligned}
& \Delta_{0}^{0}=(0,1), \quad \bar{\Delta}_{0}^{0}=[0,1], \\
& \Delta_{n}^{k}=\left(\frac{k-1}{2^{n}}, \frac{k}{2^{n}}\right), \quad \bar{\Delta}_{n}^{k}=\left[\frac{k-1}{2^{n}}, \frac{k}{2^{n}}\right], \quad(n, k) \in \Omega_{1} .
\end{aligned}
$$

Such intervals are named dyadic. Clearly, if two dyadic intervals intersect, then one of them contains the other. The inclusion $\Delta_{n}^{k} \supset \Delta_{p}^{q}$ is equivalent to conditions

$$
p \geq n, \quad 2^{p-n}(k-1)<q \leq 2^{p-n} k
$$

Put $\chi_{0}^{0}(t) \equiv 1$. If $(n, k) \in \Omega_{1}$, then

$$
\chi_{n}^{k}(x)=\left\{\begin{aligned}
& 1, t \in \overline{\Delta_{\mathrm{n}+1}^{2 \mathrm{k}-1}} \\
&-1, t \in \in \overline{\Delta_{\mathrm{n}+1}^{2 \mathrm{k}}} \\
& 0, t \in \overline{\Delta_{\mathrm{n}}^{\mathrm{k}}}
\end{aligned}\right.
$$

The value of $\chi_{n}^{k}(t)$ in a discontinuity point $t$ is defined as

$$
\chi_{n}^{k}(t)=\lim _{\varepsilon \rightarrow 0} \frac{1}{2}\left(\chi_{n}^{k}(t-\varepsilon)+\chi_{n}^{k}(t+\varepsilon)\right)
$$

If $k=1$ or $k=2^{n}$, then the value $\chi_{n}^{k}(t)$ in 0 and 1 is defined so that $\chi_{n}^{k}(t)$ is continuous in 0 and 1 .

The set of functions $\chi_{n}^{k}(t),(n, k) \in \Omega_{1}$ is called the Haar system (for convenience show by H.s.).

The one-to-one mapping of $\Omega$ on the set of integers is realized by the following:

$$
(0,0) \rightarrow 1,\left(2^{n}, k\right) \rightarrow m=2^{n}+k, \quad(n, k) \in \Omega_{1}
$$


The system $\left\{2^{n / 2}{ }_{n}^{k}(t), \quad(n, k) \in \Omega\right\}$ is orthonormal.

Let $m=2^{n}+k, \quad(n, k) \in \Omega_{1}$. Denote by $A_{m}$ the set of intervals:

$$
\begin{gathered}
A_{1}=\{(0,1)\} ; \\
A_{m}\left\{\Delta_{n+1}^{1}, \Delta_{n+1}^{2}, \ldots, \Delta_{n+1}^{2 k}, \Delta_{n}^{k+1}, \ldots, \Delta_{n}^{2^{n}}\right\}
\end{gathered}
$$

Denote by $F_{m}$ the $\sigma$-algebra generated by partitioning $A_{m}$. Clearly, each function $\left\{\chi_{i}\right\} \quad(1 \leq i \leq m)$ is measurable with respect to $F_{m}$ and $F_{m}$ is the smallest $\sigma$-algebra. Given $m \geq 1$, the polynomial $x(t)=\sum_{j=1}^{m} c_{j} \chi_{j}(t), \quad m \geq 1$

with respect to the H.s. is a step function. It is a constant on each interval from $A_{m}$. It satisfies the condition

$$
x(t)=\frac{1}{2} \lim _{\delta \rightarrow 0}(x(t-\delta)+x(t+\delta)) \quad \forall t \in(0,1)
$$

and $x(t)$ is continuous in 0 and 1 . Denote by $D_{m}$ the set of such functions.

Since $\cup_{m} D_{m}$ is dence in $L_{p}, 1 \leq p \leq \infty$, then the Haar system is a complete one in $L_{p}$.

Given $x \in L_{1}$, the Fourier - Haar coefficients are defined by

$$
\begin{aligned}
& c_{1}(x)=\int_{0}^{1} x(s) d s ; \\
& c_{m}(x)=c_{n, k}(x)=2^{n} \int_{0}^{1} x(s) x{ }_{n}^{k}(s) d s=2^{n}\left(\int_{\Delta_{n+1}^{2 k-1}} x(s) d s-\int_{\Delta_{n+1}^{2 k}} x(s) d s\right)= \\
& =2^{n}\left(\int_{\Delta_{n}}^{2 k-1} x(s)-x\left(s+2^{-n-1}\right)\right) d s,
\end{aligned}
$$

where $m=2^{n}+k, \quad(n, k) \in \Omega_{1}$.

Using standard arguments we can obtain the representation of the partial sum of Fourier - Haar series

$$
S_{m} x(t):=\sum_{i=1}^{m} c_{i}(x) X_{i}(t)=\int_{0}^{1} K_{m}(t, s) x(s) d s,
$$


where $K_{m}(t, s)=\sum_{i=1}^{m} \frac{1}{\left\|X_{i}\right\| L_{1}} X_{i}(t) X_{i}(s)$

One of the main properties of the H.s. is that it forms a basis in C, $L_{p}(1 \leq p<\infty)$. Any function $\chi_{n}(t)$ $(n>1)$ is discontinuous. Therefore if $x \in C[0,1]$, then the convergence $S_{n}(x)$ to $x$ is meant in $L_{\infty}$.

Theorem 2.1.1. If $x \in C[0,1]$, then the corresponding Haar series is convergence to the function $f$.

For proof of this Theorem see [17].

\subsubsection{Bivariate Haar systems in a special case (Tensor product in dimension two)}

Frequently, the coefficients of an everywhere convergent series $\sum_{n} a_{n} f_{n}(x)$ with respect to some orthonormal system of functions $\left\{f_{n}(x)\right\}$ are reconstructed by its sum $S(x)$ with the help of the usual Fourier formulas

$$
a_{n}=\int S(x) f_{n}(x) d s .
$$

Since the function $S(x)$ is not necessarily sum able, we assume that the integral in formula (4) is not the Lebesgue one. For example, the coefficients of an everywhere convergent trigonometric series are reconstructed with the help of the so-called MT integral ([19]).

In this section, we consider the problem of reconstruction of coefficients of the bivariate Haar series. Since there are different definitions of the (one-dimensional) Haar functions ([10]), we note that we use the standard definition ([6]) which implies that the Haar system is complete in $C[0,1]$, i.e., we assume that $X_{1}(x) \equiv 1$ on $[0,1]$; if $n=2^{k}+i, k \geq 0,1 \leq i \leq 2^{k}$, then

$$
\chi_{n}(x)=\left\{\begin{array}{l}
2^{k / 2}, x \in\left(\frac{2 i-2}{2^{k+1}}, \frac{2 i-1}{2^{k+1}}\right) \\
-2^{k / 2}, x \in\left(\frac{2 i-1}{2^{k+1}}, \frac{2 i}{2^{k+1}}\right) \\
0, x \text { not in }\left(\frac{2 i-2}{2^{k+1}}, \frac{2 i}{2^{k+1}}\right) .
\end{array}\right.
$$

We assume that at points and 1 the function $X_{n}(x)$ equals the right and left limits, respectively, and at other points of the segment $[0,1]$ it equals the arithmetic average of the right and left limits.

In [15] the p-regular convergence is considered, i. e., a convergence of the Haar series

$$
\sum_{n=1}^{\infty} \sum_{m=1}^{\infty} a_{n, m} \chi_{n, m}(x, y)=\sum_{n=1}^{\infty} \sum_{m=1}^{\infty} a_{n, m} \chi_{n}(x) \chi_{m}(y)
$$


such that the sequence of the rectangular partial sums $S_{N, M}(x, y)=\sum_{n=1 m=1}^{\infty} \sum_{n, m}^{\infty} a_{n, m}(x, y)$ verges to $S(x, y)$ as $\min (M, N) \rightarrow \infty$

and $\min (N / M, N / N) \geq p$.

\subsection{Main properties of the multivariate interpolation problem}

This section is intended to introduce multivariate polynomial interpolation.

First consider interpolation problem in univariate case. In this case, this problem has a well developed theory, see [4] and [5] for conditions ensuring its solvability.

\subsubsection{Introduction to the multivariate case}

Definition 2.2.1.1 The Lagrange interpolation problem $\left(\Pi_{n}^{k}, \chi_{s}\right)$ is called correct (poised), if for any values $\left\{c_{1}, \cdots, c_{s}\right\}$ there exists a unique polynomial $p \in \Pi_{k}^{n}$, satisfying the conditions

$$
p\left(x^{(i)}\right)=c_{i}, i=1, \ldots, s .
$$

In other words, the Lagrange interpolation problem is to find a unique polynomial

$$
p(x)=\sum_{|\gamma| \leq n} a_{\gamma} \cdot x^{\gamma} \in \Pi_{n}^{k}
$$

such that

$$
p\left(x^{(i)}\right)=\sum_{|\gamma| \leq n} a_{\gamma} \cdot\left(x^{(i)}\right)^{\gamma}=c_{i}, i=1, \ldots, s
$$

Thus, the correctness of interpolation means that the linear system (5) has a unique solution for arbitrary right hand side values. A necessary condition for this is that the number of unknowns be equal to the number of equations: i.e., $s=N$.

We know that in this case the linear system (5) has a unique solution for arbitrary values $\left\{c_{1}, \ldots, c_{s}\right\}$, if and only if the corresponding homogeneous system has only trivial solution. In other words we have

Proposition 2.2.1.2 The Lagrange pointwise interpolation problem $\left(\Pi_{n}^{k}, \chi_{N}\right)$ is correct if and only if

$$
\forall p \in \Pi_{n}^{k} \quad \text { and } \quad p\left(x^{(i)}\right)=0, i=1, \ldots, N \Rightarrow p=0 .
$$

Equivalently: The interpolation problem $\left(\Pi_{n}^{k}, \chi_{N}\right)$ is not correct if and only if 


$$
\exists p \in \Pi_{n}^{k}, p \neq 0 \text { such that } p\left(x^{(i)}\right)=0, i=1, \ldots, N
$$

When the Lagrange interpolation problem for any $N$ distinct points in $\mathbb{R}^{k}$ is poised in $V \subseteq \Pi^{k}$, then $V$ is called a Haar space of order $\mathrm{N}$.

Haar spaces exist in abundance for $k=1$. The situation for $k>1$ is dramatically different. In this case there are no Haar spaces of dimension greater than one. For refinements of this important result see $[16]$

For $k>1$, the Haar space of order $N$ of least dimension is yet to be determined and only be known for a few special cases.

\subsection{Construction of sets of interpolation points}

Since the poisedness of multivariate polynomial interpolation depends on the geometric structure of the points at which one interpolates, there has been interested in identifying points and polynomial subspaces, for example $\Pi_{n}^{k}$, for which interpolation is poised.

\subsubsection{Regular grids and natural lattices:}

Here we will focus on various methods to choose points $x_{1}, \ldots, x_{N}$ in $\mathbb{R}^{k}$ such that the interpolation problem with respect to these points is poised in $\mathbb{R}^{k}$ and moreover the Lagrange formula can be easily constructed. Clearly, this requires the following

$$
N=N_{k}=\operatorname{dim} \Pi_{n}^{k}=\left(\begin{array}{c}
\mathrm{n}+\mathrm{k} \\
\mathrm{k}
\end{array}\right) .
$$

The first and most natural approach to choose such interpolation nodes is the triangular grid of the unit simplex formed by the points in $\frac{1}{n} \mathbb{N}_{0}^{k, n}$. In the bivariate case, this configuration has been discussed in classical textbooks on numerical analysis, for example (Gasca and Sauer, 2001). This also deals with the more general case of arrays formed by points $\left(x_{i}, y_{j}\right), 0 \leq i+j \leq n$, where $x_{i}, y_{j}, i, j=$ $0, \ldots, n$, are two sets of $\mathrm{n}+1$ distinct points. A Newton formula with bivariate (tensor product) divided differences is provided for this case. The bivariate array is triangular when $x_{i}$ and $y_{j}$ are ordered and uniformly spaced.

It was this subject which apparently motivated the construction in the paper [1], written by Chung and Yao. According to [1], a set of $N$ points $X=\left\{x_{1}, \ldots, x_{N}\right\}$ in $\mathbb{R}^{k}$ satisfies the GC condition (Geometric Characterization) if for each point $x_{i}$ there exist hyperplanes $G_{i l}, l=1,2, \ldots, n$, such that $x_{i}$ is not on any of these hyperplanes, and all points of $X$ lies on at least one of them, i.e., we have

Theorem 2.2.2.1.1 let $n$ and $k$ be given. Let a set of $N$ nodes $x_{1}, \ldots, x_{N}$ be given in $\mathbb{R}^{k}$. If there exists a hyperplane $H_{i j}$ in $\mathbb{R}^{k}$, with $1 \leq i \leq N$ and $1 \leq j \leq n$, such that 


$$
x_{j} \in \cup_{v=1}^{n} H_{i v} \Leftrightarrow j \neq i,(1 \leq i \leq N)
$$

then arbitrary data on the node set with $\Pi_{n}^{k}$ is correct.

For example, let $r_{0}, r_{1}, \ldots, r_{n+1}$ be $n+2$ straight lines in $\mathbb{R}^{2}$ such that any two of them $r_{i}, r_{j}$ intersect at exactly one point $x_{i j}$ and these points have the property that

$$
x_{i j} \neq x_{k l} \Leftrightarrow\{i, j\} \neq\{k, l\}
$$

Then the set

$$
X=\left\{x_{i j}: 0 \leq i<j \leq n+1\right\}
$$

satisfies the GC condition and formula (3.3.2.1) reads as

$$
p=\sum_{i=0}^{n} \sum_{j=i+1}^{n+1} f\left(x_{i j}\right) \prod_{k=0, k \neq i, j}^{n+1} \frac{r_{k}}{r_{k}\left(x_{i j}\right)} .
$$

The set $\mathrm{X}$ is called a natural lattice of order $\mathrm{n}$.

\subsubsection{The Newton lattice}

Let a number $k \in \mathbb{Z}_{+}$and arbitrary points $a_{0}, \ldots, a_{k} \in \mathbb{R}^{k}$ be given such that $\operatorname{vol}_{k}(S) \neq 0$, where $S$ is the simplex $\left[a_{0}, \ldots, a_{k}\right]$.

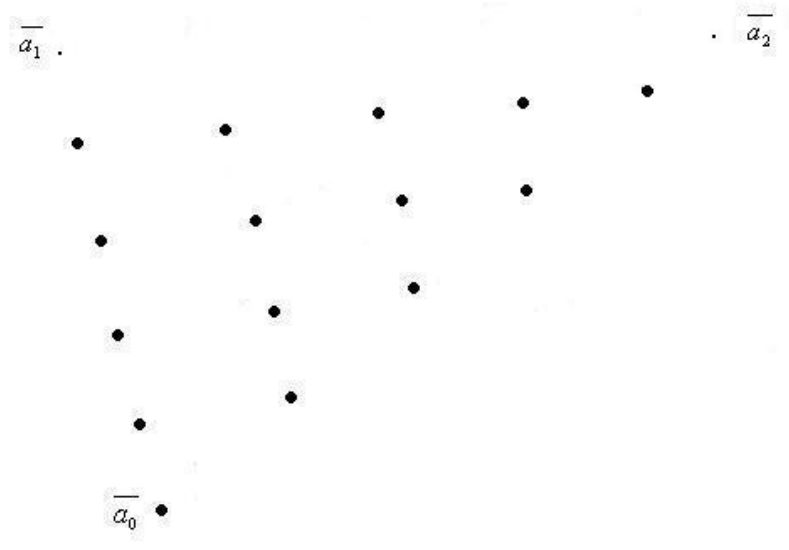

Figure 2.2.3.1: The Newton lattice $\Lambda_{N}(5)$ inside the triangle $\left[a_{0}, a_{1}, a_{2}\right]$.

It is convenient for us to introduce the Newton lattice for $\Pi_{n-1}^{k}$ inside the simplex $S$ as follows:

$$
\Lambda_{N}(n)=\left\{x_{\gamma}=a_{0}+\frac{1}{k} \sum_{i=1}^{k} \gamma_{i}\left(a_{i}-a_{0}\right): \gamma \in \Gamma\right\}
$$




$$
\text { where } \Gamma=\left\{\gamma: \gamma=\left(\gamma_{1}, \ldots, \gamma_{k}\right) \in \mathbb{Z}_{+}^{k},|\gamma| \leq n-1\right\} \text {. }
$$

\section{RESULTS AND DISCUSSION}

Now we consider two intervals same as the above interval on the plane.

Without loss of generality, let us

$$
\Lambda=\left\{(n, m): \exists k \in \mathbb{Z}_{+}, \quad n=2^{k}+i, \quad m=2^{k}+j, \quad i, j=1, \ldots, 2^{k}, k=0,1, \ldots\right\} .
$$

We only consider the problem where $(n, m) \in \Lambda$.

Denote by $\chi_{n, m}$ the Haar function of two variables in dimension two, where $m, n$ are positive integers.

Thus, by the above mentioned we have the following definitions

$$
\begin{gathered}
\chi_{1,1}(x, y)=1, x, y \in[0,1], \\
\chi_{2,2}(x, y)=\left\{\begin{array}{rr}
1, & 0 \leq x, y \leq \frac{1}{2} \\
1, & 0 \leq x \leq \frac{1}{2}, \frac{1}{2} \leq y \leq 1 \\
-1, & \frac{1}{2} \leq x \leq 1,0 \leq y \leq \frac{1}{2} \\
-1, & \frac{1}{2} \leq x, y \leq 1 .
\end{array}\right.
\end{gathered}
$$

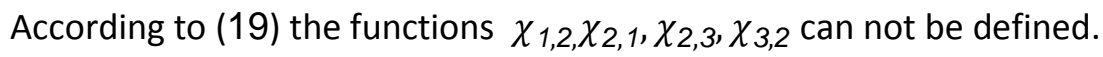

In interval $[0,1] \times[0,1]$ we also define: $\chi_{3,3}(x, y)$

\begin{tabular}{|l|l|l|l|}
\hline 0 & 0 & 0 & 0 \\
\hline 0 & 0 & 0 & 0 \\
\hline 1 & -1 & 0 & 0 \\
\hline 1 & -1 & 0 & 0 \\
\hline
\end{tabular}

$$
\chi_{3,4}(x, y)
$$


R. Dehghan, K. Rahsepar Fard / J. Math. Computer Sci. 10 (2014), 100-112

\begin{tabular}{|l|l|l|l|}
\hline 1 & -1 & 0 & 0 \\
\hline 1 & -1 & 0 & 0 \\
\hline 0 & 0 & 0 & 0 \\
\hline 0 & 0 & 0 & 0 \\
\hline
\end{tabular}

\begin{tabular}{|l|l|l|l|}
\multicolumn{1}{c}{$\chi_{4,3}(x, y)$} \\
\begin{tabular}{|l|l|l|l|}
\hline 0 & 0 & 1 & -1 \\
\hline 0 & 0 & 1 & -1 \\
\hline 0 & 0 & 0 & 0 \\
\hline 0 & 0 & 0 & 0 \\
\hline
\end{tabular}
\end{tabular}

\begin{tabular}{|l|l|l|l|}
\multicolumn{1}{c}{$\chi_{4,4}(x, y)$} \\
\begin{tabular}{|l|l|l|l|}
\hline 0 & 0 & 0 & 0 \\
\hline 0 & 0 & 0 & 0 \\
\hline 0 & 0 & 1 & -1 \\
\hline 0 & 0 & 1 & -1 \\
\hline
\end{tabular}
\end{tabular}

By this way we can define the other functions. 
Now, suppose that the function $f$ is defined on $[0,1]$. An approximation of a function $f$ is based on Haar function is as follows:

$$
f(x, y) \cong \sum_{n=1}^{M} \sum_{m=1}^{N} a_{n, m} \chi_{n, m},(20)
$$

where $M, N$ are big possitive numbers and $a_{n, m} s$ are Haar Fourier coefficient. Namely,

$$
a_{n, m}=\iint_{[0,1] \times[0,1]} f(x, y) \cdot \chi_{n, m}(x, y) d A
$$

Note that each bracket is a collection in a same power of number two.

Based on chapter two we conclude that

Theorem 3.1 Suppose $f \in C\left([0,1]^{2}\right)$ then

$$
\lim _{n, m \rightarrow \infty} \sum_{n} \sum_{m} a_{n, m} \chi_{n, m}(x, y)=f(x, y),(x, y) \in[0,1] \times[0,1] .
$$

It is easily seen that the system of $\left\{\chi_{n, m}\right\}_{n, m=1}^{\infty}$ is orthonormal, i.e.,

$$
\iint_{[0,1]^{2}} \chi_{n, m}(x, y) \cdot \chi_{p, q}(x, y) d x d y=\delta_{n, m, p, q}
$$

where $\delta_{n, m, p, q}$ is Kronecker delta.

According to (2) the function $f$ is approximated by sixth first terms, namely

$$
\begin{gathered}
f(x, y) \approx\left[a_{1,1} \chi_{1,1}(x, y)\right]+\left[a_{2,2} \chi_{2,2}(x, y)\right]+\left[a_{3,3} \chi_{3,3}(x, y)+a_{3,4} \chi_{3,4}(x, y)+a_{4,3} \chi_{4,3}(x, y)+\right. \\
a 4,4 \chi 4,4 x, y
\end{gathered}
$$

Now we bring an example of this function and approximated also by bivariate interpolation polynomial.

Example 3.2 Let $f(x, y)=\frac{1}{\left(1+x^{2}\right) \cdot\left(1+y^{2}\right)}$ be a function. Then we decide to approximate $f$ by Haar and interpolation polynomial.

Using Mathematica program the Haar Fourier coefficients are as follows:

$$
\begin{gathered}
a_{11}=\frac{\pi^{2}}{16}, \quad a_{22}=-\frac{1}{16} \pi(\pi-8 \operatorname{ArcCot}[2]), \\
a_{33}=\frac{\operatorname{ArcTan}\left[\frac{219336}{2076473}\right] \operatorname{ArcTan}\left[\frac{4}{3}\right]}{4 \sqrt{2}}, \quad a_{34}=\frac{\operatorname{ArcTan}\left[\frac{219336}{2076473}\right] \operatorname{ArcTan}\left[\frac{24}{7}\right]}{8 \sqrt{2}},
\end{gathered}
$$




$$
a_{43}=\frac{\operatorname{ArcTan}\left[\frac{1476984}{9653287}\right] \operatorname{ArcTan}\left[\frac{24}{7}\right]}{8 \sqrt{2}}, a_{44}=\frac{\operatorname{ArcTan}\left[\frac{1476984}{9653287}\right] \operatorname{ArcTan}\left[\frac{4}{3}\right]}{4 \sqrt{2}} .
$$

By approximating the above coefficients and formula (21) we have

$$
\begin{gathered}
p(x, y) \approx 0.61685 \cdot \chi_{11}(\mathrm{x}, \mathrm{y})+0.111446 \cdot \chi_{22}(\mathrm{x}, \mathrm{y})+0.0172512 \cdot \chi_{33}(\mathrm{x}, \mathrm{y})+0.0119716 \cdot \chi_{3,4}(x, y) \\
+0.0172711 \chi_{4,3}(x, y)+0.0248879 \cdot \chi_{4,4}(x, y) .
\end{gathered}
$$

Therefore

$$
p\left(\frac{1}{4}, \frac{3}{4}\right) \approx 0.740268
$$

The error is $|0.602353-0.740268|=0.137915$.

This example shows that the problem is numerically stable.

Now, for instance, consider six points

$$
(\mathbf{0}, \mathbf{0}),(a, 0),\left(a+\frac{1}{2}, \mathbf{0}\right),(\mathbf{0}, a),(a, a),\left(\mathbf{0}, a+\frac{1}{2}\right)
$$

where $0<a<\frac{1}{2}$

The above points have Newton lattice form. Thus, the interpolation polynomial coincide with these points is unique. Again by applying Mathematica program the polynomial is

$$
\begin{gathered}
p(x, y)=1+\left(-\frac{-5-14 a-7 a^{2}+6 a^{3}+8 a^{4}}{a^{3}\left(5+4 a+4 a^{2}\right)}\right) x+\left(-\frac{-5-14 a-7 a^{2}+6 a^{3}+8 a^{4}}{a^{3}\left(5+4 a+4 a^{2}\right)}\right) y \\
+\frac{2\left(-5-4 a+a^{2}+2 a^{3}\right)}{a^{3}\left(5+4 a+4 a^{2}\right)} x^{2}+\left(-\frac{2+2 a^{2}-a^{6}}{a^{4}\left(1+a^{2}\right)^{2}}\right) x * y+\frac{2\left(-5-4 a+a^{2}+2 a^{3}\right)}{a^{3}\left(5+4 a+4 a^{2}\right)} y^{2} \\
\mathrm{p}\left(\mathrm{a}, \mathrm{a}+\frac{1}{2}\right)=-\frac{10+18 a+26 a^{2}+18 a^{3}+16 a^{4}+2 a^{5}+3 a^{6}-4 a^{7}-4 a^{8}}{2 a^{3}\left(1+a^{2}\right)^{2}\left(5+4 a+4 a^{2}\right)} .
\end{gathered}
$$

If $a=\frac{1}{4}$, then

$$
\mathrm{p}\left(\frac{1}{4}, \frac{3}{4}\right)=-\frac{539726}{7225} \cong-74.7025605536332
$$


Note that this is far from to exact solution, i.e., $\frac{256}{425}=0.602353$.

This means that the problem is not stable.

\section{CONCLUSION}

In this work, the Haar wavelet approach for numerical solution of the system of interpolation problem and the system of Haar Fourier series are presented, Illustrative examples are included to demonstrate the validity and applicability of the technique. The calculations indicate that the accuracy of the obtained solutions is quite high even when the number of calculation points is small.

\section{ACKNOWLEDGEMENTS}

This study was partially supported by Islamic Azad University, Masjed Soleyman Branch. The authors are grateful for this financial support.

\section{References}

[1] K. C. Chung, and T.H. Yao, " lattices admitting unique Lagrange interpolation," SIAM J. Num. Anal 14: 735-743,1977.

[2] R. Devore, and B. Lucier, " wavelet, " Acta Numerica, 1:1-56,1992.

[3] M. Gasca, and T. Sauer, " On the history of multivariate polynomial interpolation, " Journal of Computational and Applied Mathematics, 122, 23-35,2000.

[4] M. Gasca, and T. Sauer, " Polynomial interpolation in several variables, " Advances in Computational Mathematics, $12: 377-410,2001$.

[5] L.S. Lee, and G.M. Phillips, " Polynomial interpolation at points of a geometric mesh on a triangle, " Proc. Roy. Soc. Edin. 108(A): 75-87,1988.

[6] J.R. McLaughlin, J.J Price " Comparison of Haar series with gaps with trigonometric series, " Padf. J. Math., 28( 3): 623-627,1969.

[7] M.G. Plotnikov, " Reconstruction of coefficients of the bivariate Haar series, " Russian Mathematics (Iz. VUZ), 4.9(2): 42-50,2005.

[8] M.G. Plotnikov , " The uniqueness of the everywhere convergent multiple Haar series, " Vestnik 
Mosk. Univ., Ser. Matem., 1: 23-28,2001.

[9] V.A. Skvortsov, " Differentiation with respect to meshes and the Haar series, " zametki, 4( 1):33$40,1986$.

[10] P.L . Ul'yanov, " Series by the Haar system, Matem. sbornik, "63( 3): 356-391,1964.

[11] M. Bahmanpour, and M.A. Fariborzi Araghi, " Numerical Solution of Fredholm and Volterra Integral Equations of the First Kind Using Wavelets bases" The Journal of Mathematics and Computer Science Vol .5 No.4 (2012) 337-345.

[12] S. Akhavan, " Numerical solution of singular Fredholm integro-differential equations of the second kind via Petrov-Galerkin method by using Legendre multiwavelet " Journal of mathematics and computer science 9 (2014), 321-331.

[13] A. Neamaty, B. Agheli, and R. Darzi, " Solving Fractional Partial Differential Equation by Using Wavelet Operational Method " Journal of mathematics and computer Science 7 (2013) 230 - 240.

[14] B. Bojanov,H. Hakopian H, and A.Sahakian, " Spline functions and multivariate interpolation, " Kluwer Academic Publishers, 1993.

[15] A. Cohen, " Analysis of Wavelet methods, " Elsevier, 2003.

[16] I. Novikov, and E.Semenov E, " Haar series and Linear operator, "Kluwuer Academic Publishers, 1996.

[17] B.S. Kashin, and A.A. Sahakyan, " orthogonal series, " American Mathematical Society,1996.

[18] K. Rahsepar Fard, "Orthogonal series, " Ph. D. Thesis, Yerevan State University, 2011.

[19] A. Zigmund, " Trigonometric Series, " Mir, Moscow (Russ. transl.),1965. 